\title{
Circuit
}

Musiques contemporaines

\section{Livrer un opéra, opérer un livre}

À propos de la création de Manuscrit trouvé à Saragosse de José Evangelista et Alexis Nouss

\section{Nicolas Donin}

Volume 12, numéro 2, 2002

Opéra aujourd’hui

URI : https://id.erudit.org/iderudit/902254ar

DOI : https://doi.org/10.7202/902254ar

Aller au sommaire du numéro

Éditeur(s)

Les Presses de l'Université de Montréal

ISSN

1183-1693 (imprimé)

1488-9692 (numérique)

Découvrir la revue

Citer ce document

Donin, N. (2002). Livrer un opéra, opérer un livre : à propos de la création de Manuscrit trouvé à Saragosse de José Evangelista et Alexis Nouss. Circuit, 12(2), 73-80. https://doi.org/10.7202/902254ar d'utilisation que vous pouvez consulter en ligne.

https://apropos.erudit.org/fr/usagers/politique-dutilisation/ 


\title{
CHRONIQUES
}

\section{Livrer un opéra, opérer un livre À propos de la création de Manuscrit trouvé à Saragosse de José Evangelista et Alexis Nouss}

\author{
Nicolas Donin
}

Lors d'un séjour à Montréal au mois de novembre dernier, j'ai assisté à la répétition générale (mercredi 21 ) et à la deuxième représentation (vendredi 23) de Manuscrit trouvé à Saragosse. Mon point de vue sur cette œuvre est donc borné par ces circonstances, même si ma perception a été étayée par la lecture de la partition'. Je me trouvais dans une position d'extériorité - censée justifier l'existence de ce compte rendu - assez forte, puisque vivant à Paris; à ce titre, non seulement je ne pouvais pas rattacher ce à quoi j'assistais au contexte des productions montréalaises de répertoire traditionnel (Opéra de Montréal), mais de plus mon cerveau était encore quelque peu structuré par l'actualité parisienne : je venais d'assister à Trois Sceurs de Peter Eötvös au Châtelet, et j'envisageais d'aller voir à mon retour L'Amour de loin de Kaija Saariaho (même lieu).

La commande d'un opéra à un compositeur provient de la rencontre entre sa notoriété et les intérêts d'une institution. La réalisation d'un opéra implique une somme de contraintes extrêmement importante et difficile à manipuler : c'est un métier à part entière et il ne suffit pas d'être un bon compositeur pour être un bon compositeur d'opéra. Voilà le genre de pensées qui me préoccupaient quand je suis allé découvrir Manuscrit trouvé à Saragosse. Je me demandais dans quelle mesure un compositeur pouvait se permettre de "faire comme si de rien n'était " en ne liant pas l'écriture d'un opéra à une mise en crise de son style - à défaut de
1. Je dois ici ajouter que ce texte a été écrit à la mi-décembre 2001 , c'est-à-dire avant que José Evangelista ne rédige la synthèse de son travail proposée dans le présent numéro de Circuit. 
mettre en cause l'opéra lui-même, attitude moderne qui s'est beaucoup perdue de nos jours. Or, dès les premières secondes de Manuscrit, on entrait nettement dans l'univers sonore d'Evangelista, en attendant que prenne corps celui de Potocki lu par Nouss. La modalité et l'ornementation hispanisantes de Mosquito chantant Las gitanas de Siera Morena... font signe autant vers l'Espagne du livret que vers le pays d'origine de José Evangelista. Et, très vite, I'hétérophonie se met en place, englobant les univers conjoints du chant et de l'ensemble instrumental. Tout cela aurait pu m'amener à penser que le compositeur s'était contenté de "faire du Evangelista », sans tenir compte de la singularité du médium et de la multiplicité de collaborateurs, ayant leur mot à dire, impliqués par ce médium. Mais cela n'est qu'une apparence, trompeuse car simplificatrice.

II me semble en effet que la méthode de composition propre à Evangelista a trouvé un champ d'application particulièrement pertinent dans la configuration "opéra ". La dialectique de l'hétérophonie, avec sa tension sous-jacente entre le semblable et le différent, le complémentaire et le redondant, permet de promouvoir le "beau chant" sans créer de coupure musicale entre la fosse et la scène, tout en fondant la continuité sur le primat de la mélodie ${ }^{2}$. De plus, cette technique musicale fait déjà signe vers une orientation esthétique plus large - qui sera in fine celle définie par le librettiste, dont on sait en l'occurrence l'intérêt pour le concept de métissage ${ }^{3}$. Lisons dans cette perspective le texte de synthèse où Evangelista résumait, il y a une dizaine d'années, sa théorie de la monodie. Symptomatiquement, il introduit une question de langage musical par des considérations générales sur l'enrichissement de notre compréhension de la musique par l'apprentissage de l'altérité :

La musique aujourd'hui, comme presque tous les domaines de l'activité humaine, existe à un niveau planétaire, elle n'est pas nécessairement circonscrite à une culture ou à une région particulière du monde. Ainsi, nos contacts avec les musiques d'autres cultures peuvent enrichir notre compréhension du phénomène musical. D'ailleurs, un grand nombre de compositeurs au $x x^{e}$ siècle ont reçu l'impact des musiques venues de l'extérieur du monde occidental. Or, un des traits les plus communs aux musiques d'autres cultures, spécialement celles d'Asie, est l'absence d'un langage harmonique autonome. Ce sont des musiques basées principalement sur la mélodie et sur le rythme, même dans les situations de polyphonie, c'està-dire dans la musique pour plusieurs exécutants. Dans ce cas, on emploie des techniques d'organisation verticale inusitées en Occident. La plus connue est I'hétérophonie: une ligne mélodique génère toutes les lignes d'une texture, même complexe. (Evangelista, 1991, p. 55)

La conclusion de l'article définit bien clairement le contexte où s'inscrit Evangelista, à savoir celui de l'écriture savante ${ }^{4}$, qui se trouve ainsi enrichie d'une nouvelle direction pour la pensée musicale : "L'écriture monodique, telle que décrite ici, [...] s'incorpore comme une possibilité de plus à l'intérieur de l'éventail des techniques du $x x^{e}$ siècle» (p. 70). La phrase suivante semblait appeler une expérimentation avec les conventions de l'opéra : "[l'hétérophonie] permet de redonner au paramètre mélodique un rôle prépondérant dans le discours musical :
2. À ce sujet, j'ai été étonné de lire quelques jours plus tard dans Le Devoir : "La justesse rythmique, marque de commerce du compositeur, trône au détriment de la justesse et de la gradation des effets »

(*Opéra: Promesses de métissage non tenues", Le Devoir, 24 novembre 20011. François Tousignant a pu être gêné par une musique plus evangelistienne que nature, mais dans ce cas il devrait la juger selon des critères internes : c'est bien la richesse rythmique qui donne au procédé sa valeur et son dynamisme; quant à la justesse d'intonation, qui n'était pas si déficiente, elle concerne des mélodies modales écrites dans le système tempéré, et notre oreille rétablit donc sans peine les distorsions accidentelles là moins qu'il ne s'agisse de la justesse dramatique? ce point n'est pas précisé dans la critique). Si Tousignant condamne l'emploi de l'hétérophonie en tant que telle, sous prétexte de son caractère éprouvé, il me semble qu'il passe à côté d'un des principaux intérêts de l'œuvre.

3. Voir Laplantine, F. et Nouss, A. (1997) et (2001).

4. En effet, Evangelista ne sombre jamais dans l'identification fusionnelle avec l'altérité dont il tire enseignement. Il y a là une foncière honnêteté - métissage n'est pas syncrétisme, dirait Nouss - qui peut évoquer la position de Potocki lui-même, parcourant l'Orient non en maniaque mais en noble passionné, pour reprendre ses termes dans une lettre à Adam-Kazimierz Czartoryski du début de 1805 | Enfin l'auteur de [ces lignes], qui n'a pas la manie mais la noble passion de l'Orient, Espere, qu'avec l'aide de Dieu il y aura, avec le tems à Petersbourg une tres bonne accademie orientale. [...] Et alors nous donnerons à l'Europe regulierement des nouvelles de I'Asie», Potocki [1987], p. 162). 
à la différence de la tradition occidentale des derniers siècles, la mélodie devient le seul élément unificateur du discours musical [...]» (p. 70).

Néanmoins, cette sorte de justification a posteriori de la méthode de composition d'Evangelista pour Manuscrit ne suffit pas à rendre compte des orientations musicales de l'œuvre, puisque la musique a une fonction, celle de donner vie au livret lqui peut être considéré ici comme donné antérieur, ou plutôt comme point de départ du projet - faire un opéra à partir du roman de Potocki ${ }^{5}$ ). Ici commencent les problèmes, car la structure de l'œuvre de Potocki ne se prêtait qu'au tout premier abord à la représentation théâtrale : en fait, rien de plus rebelle à la récriture ou à la réduction que cet agencement savant d'histoires successives en apparence, mais toutes reliées les unes aux autres par des jeux de renvois réciproques complexes. Face à Potocki, le librettiste et le musicien étaient obligés de s'autoréguler : toute anamnèse musicale (du leitmotiv au couper/coller) sera prise analogiquement dans le jeu littéraire des connexions entre histoires ou situations romanesques hétérogènes, à la manière du livre de Potocki pris comme totalité; par conséquent, la musique devait finir par choisir entre la lisibilité létablissement d'une typologie d'ambiances et de genres musicaux, à la manière des horizons génériques convoqués tour à tour par Potocki dans son délire baroquel et les ambiguités de la profusion. Mais du côté de l'adaptation se posait un problème symétrique : pourquoi utiliser l'œuvre de Potocki s'il s'agit de la considérer comme un roman à tiroirs traditionnels? Récrire Manuscrit pour la scène suppose de trouver un dispositif qui restitue quelque chose de la complexité vertigineuse du livre, sans devenir incompréhensible pour l'auditeur-spectateur, pris dans le moment de la représentation et dans le temps de la narration. Une enquête auprès du public pourrait sans doute nous dire si Nouss y est parvenu; pour ma part, il m'a fallu deux représentations pour comprendre les détails, mais dès la première apparaissaient simultanément l'intrigue générale (éclaircie par son retournement final) ef l'utilisation consciente d'un degré de complexité croissant - ou plutôt, s'approfondissant. J'aimerais précisément insister sur cette dernière nuance : là où le roman à tiroirs reste en général profondément linéaire lavec des stations ou des tableaux, mais sans contrevenir à la linéarité narrative), Manuscrit trouvé à Saragosse, "roman "à tiroirs gigogne" $"$ ", ouvre la dimension de la profondeur, voire du réseau souterrain (à l'image du repaire des Gomelez). Pour restituer un peu de cette profondeur tout en ouvrant les possibles de la mise en scène, Alexis Nouss a gradué les difficultés au fur et à mesure de l'histoire : alors qu'il est très facile de comprendre comment un personnage passe du discours indirect à une véritable incarnation de la scène narrée /scènes traumatiques ou hallucinations se prêtent particulièrement bien à ce mécanisme classique - voir notamment l'histoire de Pacheco, sc. 31, il est bien plus difficile de rattacher à la trame générale une histoire commençant abruptement, et faisant intervenir des personnages nouveaux dont le rapport avec ceux que l'on connaît est de plus en plus lointain et long à reconstituer ${ }^{7}-d^{\prime}$ autant qu'au fil de l'opéra, la mémoire est chargée de toujours plus d'éléments dont on ne sait s'ils seront ou non à réactiver. Au metteur en scène, troisième larron dont la place
5. On peut se référer au petit texte introductif rédigé par Alexis Nouss pour le programme des représentations : «L'idée germa d'abord dans l'esprit du compositeur. Nul étonnement à cela : Valence, d'où il est originaire, n'est pas si loin de Saragosse [...]. Il en parla au librettiste qui ne pouvait manquer, lui aussi, de se sentir concerné. Ses ascendances est-européennes le faisaient naturellement vibrer à la personnalité de Potocki [...]. Au-delà de ces particularités, tous deux étaient fascinés par ce que le roman offrait d'entrecroisement de destins et de cultures» (Nouss [2001]).

6. Selon l'expression de l'éditeur de la première version (supposée) complète de l'œuvre, René Radrizzani («Préface » à Potocki [ 1989]).

7. Je pense en particulier à la scène d'Aguilar et Tolède (sc. 15), de prime abord aussi gratuite que celle du duel - qui, elle, l'est vraiment... 
se définit ici peu à peu, d'accentuer ou non les effets de déconnexion entre histoires secrètement reliées; à lui de disséminer ou non les indices.

À lui aussi d'organiser perceptivement la densification progressive du matériau visuel impliquée par l'intrigue. En effet, lorsqu'on a fait le tour des différents chanteurs (au bout de quelques scènes), lorsqu'on a évalué la grande variété des costumes comme des usages du shôji central ou de la table, lorsqu'on a vu revenir plusieurs fois certains éléments musicaux, se pose une alternative pour l'intellection de la narration. Fautil s'appuyer sur les nouveaux costumes, toujours plus variés, et faire abstraction du retour de visages et de voix déjà rencontrées - ce qui pourrait être le simple fait d'un manque de moyens financiers pour payer une multitude de rôles secondaires? Ou bien doiton tout ramener au même en cherchant systématiquement à retrouver l'acteur derrière le rôle et, partant, en intériorisant le fait que tout cela n'est qu'illusion théâtrale? Cette alternative, qui pourrait être perçue comme une regrettable ambiguïté mal résolve par l'équipe de l'opéra, crée une tension productrice de sens puisqu'elle décrit précisément ce qui sera dénové par les dernières minutes de l'opéra (soit de la $61^{\mathrm{e}}$ à la $66^{\mathrm{e}}$ journées, dans Potocki) : toutes ces histoires n'étaient que les étapes de l'initiation de Van Worden, et les rôles secondaires étaient tous des agents des Gomelez. Par ce biais, la contrainte institutionnelle et financière (nombre limité de chanteurs) rejoint la poétique de Potocki, et permet à l'opéra de renouveler le ressort de l'intrigue avec ses moyens propres. Cette trouvaille me paraît aussi astucieuse /même si moins riche de conséquences) que le mécanisme qui permet à Eötvös, dans Trois Sœurs, d'articuler passages obligés du genre opéra et reconstruction dramatique de la pièce de Tchekhov : en recomposant trois "séquences" à partir de trois points de vue sur I'histoire (celui d'Irina, celui d'Andreï et celui de Macha), il démultiplie la temporalité de la pièce, certaines scènes étant associées seulement au personnage principal pris en référence, d'autres étant redonnées d'une séquence à l'autre mais sous un jour différent, puisque perçues par un autre protagoniste que la fois précédente; grâce à cette redistribution de la diégèse, on retrouve les alternances traditionnelles de monologues, duos, trios et scènes de groupe lavec possibilité de mettre un personnage en retrait, voire en chœur de tragédie grecquel, mais au lieu qu'elles soient justifiées par la tradition, elles le sont par une actualisation de la pièce d'origine - une refonte dramatique elle-même justifiée par la mise en opéra.

Dans Manuscrit, ce qui relie radicalisation du procédé narratif des histoires dans l'histoire et densification du matériau visuel, c'est le tourniquet des noms propres, des liens de parenté (cousines? sœurs? amantes? épouses? et bientôt mères) et des cultures (bohémiens, catholiques, juifs, musulmans : étranges étrangers qui se révéleront tous apparentés, à la façon des anneaux de Lessing). II est alors possible de comprendre tout ce que la musique est contrainte d'éviter pour que le résultat scénique soit, pourraiton dire, opératoire. La musique doit en quelque sorte se surveiller afin de ne pas faire sombrer le multiple dans le non-sens.
8. Je désigne par ce terme propre à l'architecture intérieure japonaise le panneau coulissant en papier translucide dont certaines parties pouvaient être ouvertes au gré des décors auxquels il sert de substitut. 
La science du divers construite par la machine littéraire potockienne ${ }^{9}$ ne peut être exaltée qu'à condition de renforcer par ailleurs les facteurs unifiants.

Question de cadre culturel : la typologie traditionnelle des voix n'est pas remise en cause, contrairement à ce que fait Eötvös ${ }^{10}$ dans Trois Sœurs, pour reprendre cet exemple. Mais par ailleurs la musique obéit à un découpage très clair par numéros (ainsi, il n'y a pas de jeux de chevauchement, ou de tuilage, entre scènes successives et accompagnement musicall) et établit des caractères musicaux assez rigides allant du pastiche dix-huitième siècle (pour le duel) au plus pur idiome evangelistien, en passant par les imitations de tournures folkloriques et par les citations littérales (la prière en hébreu). II faut bien prendre en compte ici la nécessité musicale de donner du fixe, du repérable, de l'immédiat, là où la diégèse ne cesse de se renouveler et se complexifier. À cet égard, la charge proprement musicale du livre de Potocki, souvent soulignée par ses exégètes consciemment ou inconsciemment $^{\prime \prime}$, doit être encore une fois considérée comme un donné de départ auquel le compositeur devait s'adapter en évitant la redondance; ainsi, après avoir employé une métaphore musicale ${ }^{12}$, René Radrizzani en vient à caractériser les dernières journées comme une "impressionnante [...] strette finale ${ }^{13}$ » : l'enjeu du passage à l'opéra n'était-il pas alors de prendre une saine distance avec les modèles wagnérien et beethovénien qui auraient incité le compositeur à redoubler la musicalité du livret par une polyphonie narrative (leitmotive) et une polyphonie simultanée (finale en fugato)?

Autre présence musicale dans le livre de Potocki, celle de la mise en scène d'opéra. "Bon nombre de [nos] effets scéniques renvoyaient ouvertement aux conventions de l'opéra, entendues au sens large. II paraît d'ailleurs évident que Potocki a tiré plus d'un effet "gothique" du Manuscrit des visites qu'il avait pu faire dans différents opéras de l'époque ${ }^{14}$ ", écrit Tadeusz Bradecki, au sujet de l'adaptation théâtrale de l'œuvre qu'il avait donnée au Stary Teatr (Cracovie) en 1992. De leur côté, Nouss et Laplantine en venaient déjà, en analysant des cas de métissages, à évoquer l'opéra baroque :

La naissance et le développement de l'opéra au XVII siècle sont indissociables de l'art baroque dont il est le genre musical par excellence. Or le baroque pratique le métissage comme une seconde nature [...]. L'opéra [... ] jove sur le partage, la juxtaposition ou l'empiètement des espaces visuels (coulisses, scène et fosse) et sonores (chanteurs/orchestre et chanté/parlé) 15 .

Pourtant les possibilités de mise en abîme, d'évocation de l'opéra dans l'opéra, qui auraient pu remplacer la problématique - interne au Manuscrit en tant que fait littéraire - de l'illusion théâtrale et du mensonge romanesque ${ }^{16}$, n'ont pas été exploitées. À cet égard, il semble que le metteur en scène se soit parfois comporté vis-à-vis de l'opéra comme Potocki rentrant en Pologne (avril 1788) et déclarant : "Je connois peu mon Paiis; mais je connais les autres : Je ferai des comparaisons, et j'ose deja promettre des resultats consolants ${ }^{17}$ \%. Wajdi Mouawad n'hésite pas
9. Voir à ce sujet le Potocki épistémologue de l'histoire et anthropologue avant la lettre décrit par Nicolas Hafid-Martin : «[Potocki] introduit intuitivement la complexité au nombre des caractéristiques humaines. Qu'il s'agisse d'une nation ou d'un individu, chaque ensemble ne peut être défini que par la somme de ses nombreuses composantes, fussentelles contradictoires. " Et: " Le monde n'est que le chatoiement des rapports enchevêtrés entre les choses et nous. La relation d'un périple doit par conséquent refléter cet universel mouvement pour être véridique " (Hafid-Martin [2001], p. 53).

10. Tous les rôles sont chantés par des hommes (les trois sœurs sont des contreténors).

11. Ainsi François Rosset au sujet de la structure du livre: "Ces deux rythmes, celui du voyage et celui de la narration se superposent, mais ni systématiquement, ni schématiquement " (Rosset [2001], p. 87).

12. "Le Manuscrit trouvé à Saragosse est une vaste composition polyphonique, dans laquelle chaque protagoniste a, dans son registre particulier, une voix indépendante, reliée aux autres et à l'ensemble par le jeu des rencontres et des leitmotive.

Musicalement parlant, il s'agit d'une grande fugue " («Préface» à Polocki [1989], p. XIII).

13. Ibid, p. XIII.

14. Bradecki, p. 182.
15. Laplantine et Nouss (1997), p. 51-52. 16. Cf. Rosset (1991), p. $110-111$ et
p. $116-117$.

17. «Essai de logique de Jean Potocki », dans : Potocki (1987). 
à transférer des schémas théâtraux qui mettent en péril la discipline corporelle des chanteurs (chanter allongé la tête en bas, par exemple) mais ne pousse pas toujours assez loin dans cette direction /d'où le statisme en déshérence de la première scène et l'inefficacité de la scène où Camille et Inésille poursuivent Pacheco), ou ne résout pas des effets visuels suggestifs (quel sens prend la manipulation verticale et oblique de la table/autel des plaisirs? à quoi rattacher le ridicule de la scène du duel [à commencer par les costumes], dans un contexte où l'honneur est si valorisé qu'il est ce qui donne à la trajectoire du héros sa cohérence et son dynamisme?).

Si les auteurs de Manuscrit trouvé à Saragosse avaient entièrement réussi leur coup, je n'aurais sans doute pas été tenté d'expliquer leur travail à partir de ce qu'il évite, de ce qu'il n'est pas, des contraintes qu'il dépasse. Néanmoins, il est du ressort du travail critique de montrer l'artifice là où tout semble aller de soi. J'ai vu l'opéra fonctionner sur le public, j'ai vu les chanteurs s'investir dans le monde divers et consistant auquel ils étaient chargé de donner vie. II me semble que ce ne sont pas ses seules raisons d'être (en dehors des mobiles extra-artistiques), puisqu'il parvient à donner sens à la question transverse du métissage sur plusieurs plans : celui du récit adapté ${ }^{18}$, celui de l'intégration du divers et de l'étranger dans un langage musical, celui, enfin, du genre de l'opéra let du problème de son unitél. En nouant ces plans, Manuscrit trouvé à Saragosse trouve une certaine cohérence esthétique. Cela implique un surcroît de contraintes qui n'ont pu être résolues en quelques jours ou semaines de mise en place; aussi ne peut-on pas, selon moi, statuer de façon péremptoire sur la qualité de l'opéra, ni sur la consistance de la problématique du métissage, comme l'ont fait les critiques montréalais lors d'une réaction à chaud qui prenait très peu en compte les présupposés artistiques de l'entreprise et les dangers auxquels elle faisait face.
18. La pratique du récit serait, dans l'esthétique du librettiste, du même côté que le phénomène musical : « Le métissage est [...] certainement plus auditif que visuel, plus musical que pictural. [...] De la même manière, le récit convient beaucoup mieux pour rendre compte du métissage que la description 》 (Laplantine et Nouss [1997], p. 83).

\section{Bibliographie}

BRADECKI, T. (2001), "Le Manuscrit théâtral ", Europe n 863, mars 2001 ("Jean Potocki / Littérature et enseignement»), p. 177-185.

EVANGELISTA, J. (1991), "Pourquoi composer de la musique monodique», Circuit, vol. $1, n^{\circ} 2$, p. 55-70.

HAFID-MARTIN, N. (2001), "Voyage et connaissance», Europe n 863, mars 2001 ["Jean Potocki / Littérature et enseignement»), p. 48-69.

LAPLANTINE, F. et NOUSS, A. (1997), Le Métissage, Paris, Flammarion, 114 p.

LAPLANTINE, F. et NOUSS, A. (2001), Métissages d'Arcimboldo à Zombi, Paris, Pauvert, $636 \mathrm{p}$. 
NOUSS, A. (2001), "Opéra trouvé à Montréal », programme de la création de Manuscrit trouvé à Saragosse, Montréal, Chants Libres / SMCQ, $2^{\mathrm{e}}$ de couv.

POTOCKI, J. [TRIAIRE, D. (dir.)] (1987), Jean Potocki. Écrits politiques, Paris, Honoré Champion, $335 \mathrm{p}$.

POTOCKI, J. [RADRIZZANI, R. (dir.)] (1989), Manuscrit trouvé à Saragosse, Paris, José Corti, $680 \mathrm{p}$.

ROSSET, F. (1991), Le Théâtre du romanesque: "Manuscrit trouvé à Saragosse" de Jean Potocki, entre construction et maçonnerie, Lausanne, l'Âge d'Homme, $267 \mathrm{p}$.

ROSSET, F. (2001), "Introduction au Manuscrit trouvé à Saragosse", Europe $n^{\circ} 863$, mars 2001 ("Jean Potocki / Littérature et enseignement»), p. 84-90. 


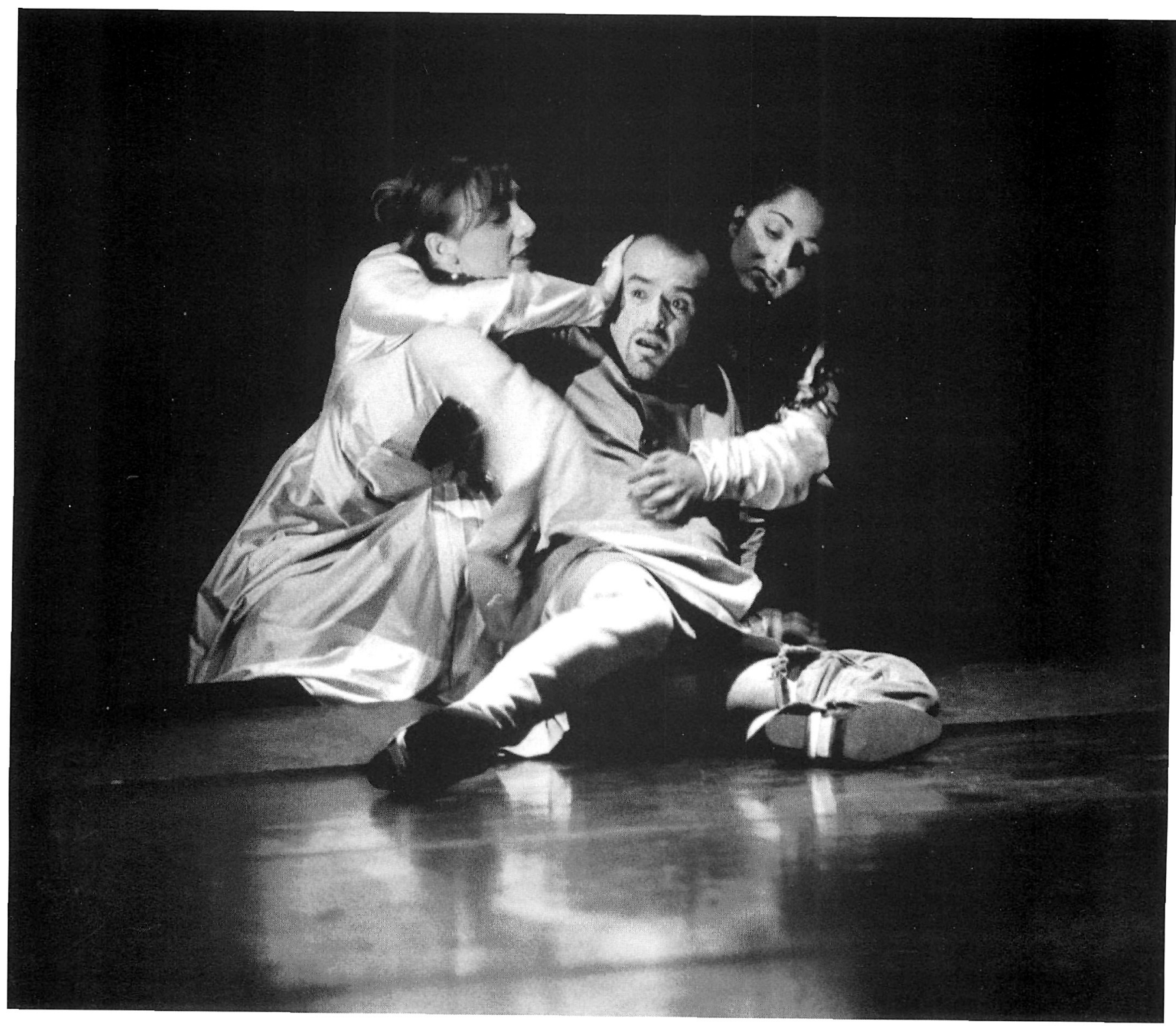

